\section{A case series review of patients with Thrombocytopenia and Absent- Radii syndrome (TARS) and their management during pregnancy}

\author{
Daniel Halperin ${ }^{1 *}$, Sue Pavord ${ }^{2}$ and Bethan Myers ${ }^{3}$ \\ 'Specialist Registrar, Haematology, Leicester Royal Infirmary, UK \\ ${ }^{2}$ Consultant Haematologist, Oxford University Hospitals, UK \\ ${ }^{3}$ Consultant, Haematology, Leicester Royal Infirmary, UK
}

\section{Abstract}

Bleeding diatheses due to platelet-related disorders can present challenges to treating clinicians especially in the context of peri- and post-partum patients in the obstetric setting. TARS is an inherited disorder characterised by reduced bone marrow platelet production, skeletal deformities affecting radii and other limbs; cardiac, renal, and other heterogeneous anomalies may occur. It is caused by co-inheritance of a microdeletion and a nucleotide polymorphism in the RBM8A gene on chromosome 1.

Bleeding phenotype is more severe than platelet numbers might predict especially in infants but improves with age. There is minimal literature regarding impact in pregnancy and puerperium.

We describe management of three pregnancies in the haematology-obstetrics clinic. As platelet counts normally decrease through pregnancy, close monitoring is required in TAR syndrome. No major bleeding was seen antenatally but two required platelet transfusion during labour. No other treatment definitely improves bleeding, although case reports of steroids claim variable success.

Tranexamic acid may be helpful, and thrombopoietin agonists represent a potential future option.
More Information

*Address for Correspondence: Daniel Halperin Department of Medical Education, Jarvis Building Level 2, Leicester Royal Infirmary, Infirmary Square, Leicester LE1 5WW, UK,

Email: d.halperin@nhs.net; daniel.halperin@uhl-tr.nhs.uk

Submitted: 30 July 2020

Approved: 11 August 2020

Published: 12 August 2020

How to cite this article: Halperin D, Pavord $S$, Myers B. A case series review of patients with Thrombocytopenia and Absent-Radii syndrome (TARS) and their management during pregnancy. Clin J Obstet Gynecol. 2020; 3 : 090-092.

DOI: 10.29328/journal.cjog. 1001056

Copyright: @ 2020 Halperin D, et al. This is an open access article distributed under the Creative Commons Attribution License, which permits unrestricted use, distribution, and reproduction in any medium, provided the original work is properly cited.

(W) Check for updates OPEN ACCESS

\section{Case reports}

\section{Case 1}

A 24 year-old woman was referred in her sixth pregnancy with platelet count of $73 \times 10^{9} / \mathrm{L}$, (history of 3 miscarriages, 2 termination of pregnancies). Her only bleeding occurred after orthopaedic surgery, requiring blood and platelet transfusions. She remained asymptomatic throughout pregnancy. Her platelet count fell to $40 \times 10^{9} / \mathrm{L}$ at term. Platelet transfusion was not required, and she had a normal vaginal delivery. Standard measures to minimise trauma to the neonate were instituted, and cord blood platelet count was $201 \times 10^{9} / \mathrm{L}$. Postnatally, there was no excessive bleeding, and 6 weeks later platelet count was 101.

\section{Case 2}

A 23 year-old woman was referred at 6 weeks' gestation following IVF. She had partial TARS with one radius affected. Her platelet count had varied, with occasional drops to single figures, duringinfections. Booking plateletcount was $94 \times 10^{9} / \mathrm{L}$. Scans at 16 weeks demonstrated normal radii in the foetus. Her platelet count fell progressively to a level of $31 \times 10^{9} / \mathrm{L}$ by 26 weeks, then remaining stable to delivery. A delivery plan outlined precautions for an atraumatic birth, platelet transfusion was given at delivery and third stage of labour was active managed with intravenous syntocinon.

\section{Case 3}

A 32 year-old woman with TARS and seronegative arthritis was referred at 21 weeks' gestation in her first pregnancy. Baseline platelet count was 70-110 x 10\% $/$ L. Booking platelet count was $73 \times 10^{9} / \mathrm{L}$ decreasing to $48 \times 10^{9} / \mathrm{L}$ at $28 \mathrm{w}$. Detailed scan at 20 weeks showed no foetal abnormalities.

She was monitored 4-weekly and just prior to delivery 
had dropped further to $26 \times 10^{9} /$ L. Delivery was by elective caesarean at 38 weeks due to breech presentation, covered with two pools of platelets with no abnormal bleeding either peri- or postpartum.

Five years later she booked at $13 \mathrm{w}$, with platelet count of $84 \times 10^{9} / \mathrm{L}$, falling to $44 \times 10^{9} / \mathrm{L}$ by 26 weeks. Unfortunately, the patient contracted streptococcal pneumonia and prematurely ruptured her membranes at $30+3$ weeks, complicated by antenatal haemorrhage and placental abruption necessitating emergency lower segment caesarean section under general anaesthetic. Blood loss was $1100 \mathrm{mls}$ treated with platelet transfusions and tranexamic acid.

\section{Discussion}

Bone marrow examination in TARS demonstrates low megakaryocyte numbers with preservation of other lineages. Ballmaier, et al. [1] reported elevation of thrombopoietin in all patients, suggesting reduced receptor sensitivity to TPO. Bleeding phenotype appears out of proportion to platelet count.

Infants have bleeding problems, but platelet count rises rapidly within the first years, reaching nearnormal levels in adulthood; exacerbations occur due to various stresses, infection, and diet in addition to pregnancy. Patients often have associated cardiac, renal, and joint problems, and cow's milk intolerance. Limb abnormalities may be simple radial aplasia or involve skeletal structure akin to thalidomide. The radial artery is preserved suggesting primary aplasia rather than vascular insufficiency. Skeletal effects are also commonly observed in the legs and feet [2]. Occasionally affected females have urogenital anomalies such as absent uterus [3].

The condition was originally described as autosomal recessive, but more recent work by Klopocki, et al. [4] suggested TARS does not fit into standard inheritance pattern on a single gene, and a microdeletion on chromosome 1q21.1 was found in all cases. As also present in 32\% unaffected family members, this suggests that it is necessary but not sufficient to cause the syndrome. Further studies demonstrate that nearly all TARS patients carry single nucleotide polymorphisms in one allele of the RBM8A gene, coded in the 1q21.1 region.

The hypothesis is that one causative factor is inherited from each parent i.e. compound inheritance of a null allele and specific SNP (in the majority of cases). Mutations in other genes have been found as part of a wider spectrum of symptoms but may represent other diseases.

There is little in the medical literature about TARS in pregnancy and its management. In our cases, all 3 showed a marked reduction in platelet count during pregnancy but with no bleeding complications for the first two. Bleeding in the third case was more likely obstetric rather than due to thrombocytopenia.
However, TARS may present problems for obstetric anaesthesia. Thrombocytopenia may prevent safe neuraxial anaesthesia. The platelet transfusion guidelines from the British Society of Haematology [5] recommend a minimum platelet count of $80 \times 10^{\circ} / \mathrm{L}$, although lower thresholds may be acceptable in selected patients [6]. Platelet transfusion may be required before neuraxial anaesthetic, with confirmation of an acceptable platelet count post transfusion. Consider the possibility of HLA antibodies in pre-transfused patients as availability of matched platelets can take several hours.

Vascular access may be difficult depending on degree of upper limb deformity; brachial access may even be impossible. Repeated use of veins e.g. for platelet transfusions can also present challenges. Upper limb arterial BP monitoring may be difficult, with alternative sites needed.

Other issues include micrognathia, present in 50\% cases, which may present difficulty in intubation, as well as cardiac and renal abnormalities, which should be assessed antenatally and prior to delivery.

Two of the women had difficulty conceiving, suggesting a link between TARS and reduced fertility.

Reassuringly, all were managed with platelet transfusions and did not require extreme measures. As a disorder of platelet production there are few therapeutic strategies to improve the count beyond transfusion. Medications which affect platelet function should be used cautiously, with riskbenefit ratio of aspirin for prevention of pre-eclampsia and increased bleeding risk. Postpartum use of NSAIDs should be avoided. Tranexamic acid and desmopressin have been used as adjunctive treatment for bleeding in patients with TARS.

There is one case report of recombinant factor VIIa use in planned surgery in a patient with a bleeding phenotype, however this unlicensed use of the product is not recommended by the company. Finally, whilst there is no published evidence at the current time, thrombopoietin receptor agonists may represent potential therapeutic options. There are reports of these agents used in pregnancy for women with refractory ITP, although no data for use in TARS as yet.

\section{Contributorship}

BM planned the case series. All 3 authors contributed to the body of text. DH wrote the initial draft with both BM and SP adding content as well as revisions.

\section{References}

1. Ballmaier M, Schulze H, Strauss G, Cherkaoui K, Wittner N, et al Thrombopoietin in patients with congenital thrombocytopenia and absent radii: elevated serum levels, normal receptor expression, but defective reactivity to thrombopoietin Blood. 1997; 90: 612-619. PubMed: https://pubmed.ncbi.nlm.nih.gov/9226161/

2. Greenhalgh KL, Howell RT, Bottani A, Ancliff PJ, Brunner HG, et al Thrombocytopenia-absent radius syndrome: a clinical genetic study. $J$ 
Med Genet. 2002; 39: 876-881.

PubMed: https://pubmed.ncbi.nlm.nih.gov/12471199/

3. Hall JG, Levin J, Kuhn JP, Ottenheimer EJ, van Berkum KA, et al Thrombocytopenia with absent radius (TAR). Medicine (Baltimore). 1969; 48: 411-439.

PubMed: https://pubmed.ncbi.nlm.nih.gov/4951233/

4. Klopocki E, Schulze H, Strauss G, Ott CE, Hall J, et al. Complex inheritance pattern resembling autosomal recessive inheritance involving a microdeletion in thrombocytopenia-absent radius syndrome.
Am J Hum Genet. 2007; 80: 232-2400.

PubMed: https://pubmed.ncbi.nlm.nih.gov/17236129/

5. Estcourt LJ, Birchall J, Allard S, Bassey SJ, Hersey P, et al. Guidelines for the use of platelet transfusions. Br J Haematol. 2017: 176; 365-394. PubMed: https://pubmed.ncbi.nlm.nih.gov/28009056/

6. Hunt BJ, Lyons G. Platelet Counts and Obstetric Analgesia and Anaesthesia Prepared by: Gordon Lyons FRCA MD Beverley J Hunt, FRCP, FRCPath, MD Obstetric Anaesthesia Professor of Thrombosis \& St James' University Hospital Haemostasis. 2017. 